\title{
Hadron physics at KLOE: results and prospects
}

\author{
Paolo Gauzzi* for the KLOE Collaboration ${ }^{\dagger}$ \\ Dipartimento di Fisica, Sapienza Università di Roma e INFN Sezione di Roma, P.le A.Moro 2, \\ 00185 Rome (Italy) \\ E-mail: Paolo.Gauzzi@roma1.infn.it
}

KLOE collected $2.5 \mathrm{fb}^{-1}$ of data at the peak of the $\phi(1020)$ resonance at the $e^{+} e^{-}$collider DAФNE. About $10^{8} \eta$ mesons have been produced through the radiative decay $\phi \rightarrow \eta \gamma$. Results on the decay channels $\eta \rightarrow 3 \pi^{0}, \eta \rightarrow \pi^{+} \pi^{-} \gamma$ and $\eta \rightarrow \pi^{+} \pi^{-} e^{+} e^{-}$are presented.

Using the $250 \mathrm{pb}^{-1}$ collected off-peak at $\sqrt{s}=1 \mathrm{GeV}$ the $e^{+} e^{-} \rightarrow e^{+} e^{-} \gamma \gamma \rightarrow e^{+} e^{-} \pi^{0} \pi^{0}$ process has been studied looking for the contribution of the $\sigma(600)$, and the cross-section of $e^{+} e^{-} \rightarrow e^{+} e^{-} \gamma \gamma \rightarrow e^{+} e^{-} \eta$ has been measured.

The latest results on the hadronic cross-section measurement that confirm the three standard deviation discrepancy between the experimental and the Standard Model values of the muon magnetic anomaly are reported.

Some prospects for the new data-taking of KLOE starting at the beginning of 2011 at the upgraded DAФNE are also discussed.

35th International Conference of High Energy Physics - ICHEP2010,

July 22-28, 2010

Paris France

\footnotetext{
${ }^{*}$ Speaker.

${ }^{\dagger}$ The KLOE Collaboration: F. Ambrosino, A. Antonelli, M. Antonelli, F. Archilli, G. Bencivenni, C. Bini, C. Bloise, S. Bocchetta, F. Bossi, P. Branchini, G. Capon, T. Capussela, F. Ceradini, P. Ciambrone, E. De Lucia, A. De Santis, P. De Simone, G. De Zorzi, A. Denig, A. Di Domenico, C. Di Donato, B. Di Micco, M. Dreucci, G. Felici, S. Fiore, P. Franzini, C. Gatti, P. Gauzzi, S. Giovannella, E. Graziani, M. Jacewicz, J. Lee-Franzini, M. Martini, P. Massarotti, S. Meola, S. Miscetti, M. Moulson, S. Müller, F. Murtas, M. Napolitano, F. Nguyen, M. Palutan, A. Passeri, V. Patera, P. Santangelo, B. Sciascia, T. Spadaro, C. Taccini, L. Tortora, P. Valente, G. Venanzoni, R.Versaci, G. Xu
} 


\section{Introduction}

KLOE is a multipurpose detector, consisting of a large cylindrical drift chamber with an internal radius of $25 \mathrm{~cm}$ and an external one of $2 \mathrm{~m}$, surrounded by a lead-scintillating fibers electromagnetic calorimeter, which provides a $98 \%$ solid angle coverage. Both are immersed in the 0.52 $\mathrm{T}$ field of a superconducting solenoid. From 2000 to $2006 \mathrm{KLOE}$ has collected $2.5 \mathrm{fb}^{-1}$ of data at the peak of the $\phi(1020)$ at the $e^{+} e^{-}$collider DAФNE in Frascati, plus $250 \mathrm{pb}^{-1}$ off-peak.

In this paper the recent KLOE results on $\eta$ decays and on the physics of the continuum ( $\gamma \gamma$ processes and hadronic cross-section) are presented.

During 2008 a new interaction scheme of the DAФNE $\phi$-factory has been succesfully tested, reaching a peak luminosity of about a factor of three larger than what previously obtained. Following these achievement, a new data-taking with an improved detector will start at the beginning of 2011 (referred to as KLOE-2 experiment). The KLOE-2 present upgrade consists in the installation of two different $e^{ \pm}$taggers for $\gamma \gamma$ physics: the Low Energy Tagger, made of two crystal calorimeters placed very near the DAФNE Interaction Point (IP), and the High Energy Tagger, made of two position sensitive detectors placed far from the IP, after the first bending dipoles of DA $\Phi$ NE. After the collection of about $5 \mathrm{pb}^{-1}$ in one year data-taking, a major detector upgrade is planned[1], aiming to collect about $20 \mathrm{pb}^{-1}$ of data to complete the KLOE physics program[2].

\section{2. $\eta$ decays}

The properties of the $\eta$ meson can be studied through the radiative decay $\phi \rightarrow \eta \gamma$. The full KLOE data set corresponds to about $10^{8} \eta$ produced. With this sample the dominant decay modes like $\eta \rightarrow 3 \pi$ as well as the rare ones $\eta \rightarrow \pi^{+} \pi^{-} \gamma$ and $\eta \rightarrow \pi^{+} \pi^{-} e^{+} e^{-}$have been studied.

$\eta \rightarrow \pi^{0} \pi^{0} \pi^{0}$. The three-pion decay is isospin-violating and is due to a term proportional to the $d$ and $u$ quark mass difference in the QCD Lagrangian, thus is suitable for a precise determination of the ratios of the light quark masses. The Dalitz plot of the neutral decay $\eta \rightarrow 3 \pi^{0}$ is symmetric and its density is described by the slope parameter $\alpha,|A(Z)|^{2} \propto 1+2 \alpha Z$, where $Z=(2 / 3) \sum_{i=1}^{3}\left[\left(3 E_{i}-m_{\eta}\right) /\left(m_{\eta}-3_{\pi}\right)\right]^{2}$. From a sample of $450 \mathrm{pb}^{-1}, 6.5 \times 10^{5}$ events with seven prompt photons have been selected with negligible background. From the fit of the $Z$ distribution $\alpha=-0.0301 \pm 0.0035_{-0.0036}^{+0.0022}$ has been obtained[4], consistent with the previous experimental determinations, but in disagreement with Chiral Perturbation Theory $(\chi \mathrm{PT})$ calculations at NNLO which predict a positive value[3].

$\eta \rightarrow \pi^{+} \pi^{-} \gamma$. A significant contribution to the amplitude of $\eta \rightarrow \pi^{+} \pi^{-} \gamma$ is expected from the box anomaly, which is a higher order term of the Wess-Zumino-Witten Lagrangian. The two pion invariant mass distribution can be used to disentangle this contribution from the resonant ones, dominated by the $\rho$ meson. In this respect the existing measurements done in the 1970s are not sufficient for an unambiguous interpretation[5]. Moreover the most recent determination of the branching ratio by the CLEO Collaboration is two standard deviation lower than the older ones.

From the analysis of a sample of $1.2 \mathrm{fb}^{-1}$ of data, a measurement of the branching ratio normalized to $\eta \rightarrow \pi^{+} \pi^{-} \pi^{0}$ has been obtained, $\Gamma\left(\eta \rightarrow \pi^{+} \pi^{-} \gamma\right) / \Gamma\left(\eta \rightarrow \pi^{+} \pi^{-} \pi^{0}\right)=0.201 \pm 0.006$ in good agreement with the older measurements. The uncertainty is dominated by systematics. Work is in 

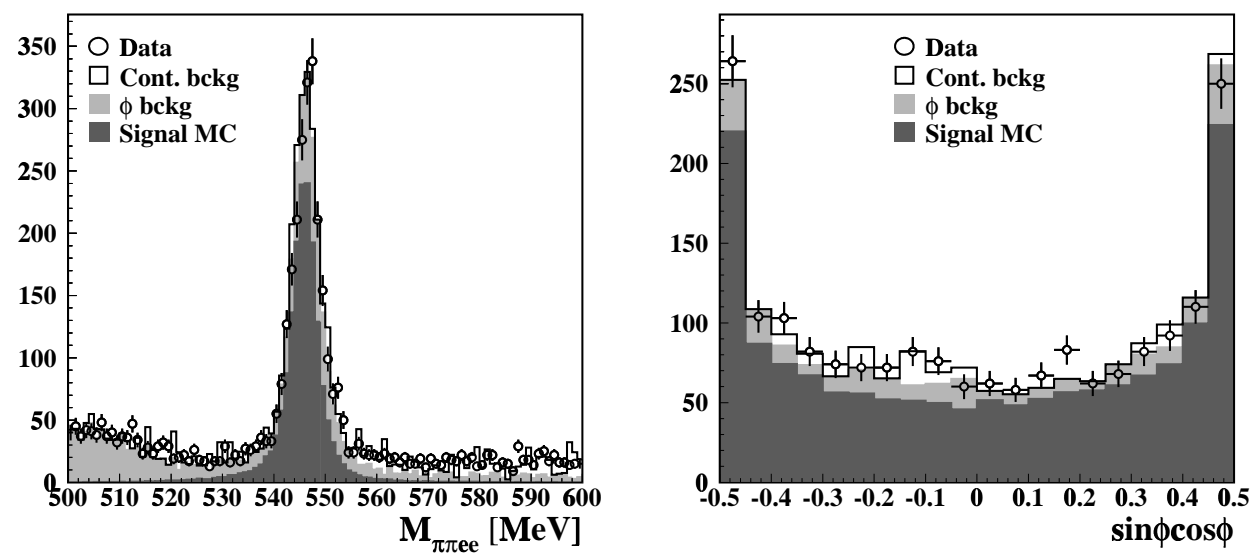

Figure 1: Left: $\pi^{+} \pi^{-} e^{+} e^{-}$invariant mass distribution. Right: distribution of $\sin \phi \cos \phi$ variable (see text for the $\phi$ angle definition).

progress to analyze the whole KLOE data sample, with the aim to reduce the total uncertainty to about $1 \%$, and to study the $\pi^{+} \pi^{-}$invariant mass distribution.

In the first year of the KLOE-2 data-taking we expect to collect about $10^{5} \eta^{\prime} \rightarrow \pi^{+} \pi^{-} \gamma$; this will allow a combined analysis of the box anomaly in both $\eta$ and $\eta^{\prime}$ decays.

$\eta \rightarrow \pi^{+} \pi^{-} e^{+} e^{-}$. The conversion decay $\eta \rightarrow \pi^{+} \pi^{-} \gamma^{*} \rightarrow \pi^{+} \pi^{-} e^{+} e^{-}$allows to probe the internal structure of the $\eta$ meson measuring the virtual photon 4-momentum via the invariant mass of the lepton pair. The theoretical calculation of the branching ratio ranges from $25 \times 10^{-5}$ to $36 \times 10^{-5}$ and the previous exprimental measurements are based on very low statistics samples, $\operatorname{Br}(\eta \rightarrow$ $\left.\pi^{+} \pi^{-} e^{+} e^{-}\right)=\left(37_{-18}^{+25} \pm 30\right) \times 10^{-5}$ (4 events) by CMD-2 and $B r\left(\eta \rightarrow \pi^{+} \pi^{-} e^{+} e^{-}\right)=\left(43_{-16}^{+20} \pm\right.$ $4) \times 10^{-5}$ (16 events) by the WASA experiment at CELSIUS.

An asymmetry in the distribution of the angle $\phi$ between the decay planes of the leptons and of the pions, would be a signal of CP violation. This asymmetry is constrained by experimental and Standard Model upper limits on the CP violating decay $\eta \rightarrow \pi^{+} \pi^{-}$, to be at most of $O\left(10^{-4}\right)$ and $O\left(10^{-15}\right)$ respectively. However a possible CP violation mechanism beyond the Standard Model that can raise the asymmetry up to $2 \times 10^{-2}$ has been proposed[6].

In a sample of $1.73 \mathrm{fb}^{-1}, 1555 \pm 52$ signal events with a background of 368 have been selected (see fig.2), from which $\operatorname{Br}\left(\eta \rightarrow \pi^{+} \pi^{-} e^{+} e^{-}(\gamma)\right)=(26.8 \pm 0.9 \pm 0.7) \times 10^{-5}$ has been obtained[7]. The plane asymmetry has been measured for the first time, $A_{\phi}=(-0.6 \pm 2.5 \pm 1.8) \times 10^{-2}$.

With a sample of $20 \mathrm{fb}^{-1}$ and the increase of the efficiency expected with the insertion of an Inner Tracker[1], KLOE-2 could improve the precision on $A_{\phi}$ at the level of $1 \%$.

\section{3. $\gamma \gamma$ physics}

In $\gamma \gamma$ processes, like $e^{+} e^{-} \rightarrow e^{+} e^{-} \gamma^{*} \gamma^{*} \rightarrow e^{+} e^{-} X, C=+1$ hadronic states can be produced. If both photons are quasi-real, the Equivalent Photon Approximation can be done and the event yield can be evaluated according to $N_{e e X}=L \int \frac{d F}{d W_{\gamma \gamma}} \sigma_{\gamma \gamma \rightarrow X}\left(W_{\gamma \gamma}\right) d W_{\gamma \gamma}$, where $W_{\gamma \gamma}$ is the two photon invariant mass, $L$ is the integrated luminosity and $d F / d W_{\gamma \gamma}$ is the $\gamma \gamma$ flux function. As shown in 
fig. 3 at the DAФNE energy the final states with a the single $\pi^{0}$ or $\eta$ as well as the the two pion one can be detected. The latter is interesting for the study of the $\sigma(600)$ scalar meson, via the reaction $\gamma \gamma \rightarrow \sigma(600) \rightarrow \pi \pi$. The cleanest channel is $\gamma \gamma \rightarrow \pi^{0} \pi^{0}$; about $240 \mathrm{pb}^{-1}$ of data collected off-peak at $\sqrt{E}=1 \mathrm{GeV}$, to reduce the large background from $\phi$ decays, have been analyzed. Events with only four prompt photons have been selected, the scattered leptons are not detected as they escape in the DAФNE beam-pine. In fig. 3 the distribution of the four photon invariant mass is shown; the residual background has been evaluated according to the expected cross-sections of the various processes. Work is in progress to check the background normaliation and to extract the $\gamma \gamma \rightarrow \pi^{0} \pi^{0}$ cross-section.

Using the same data the $\eta$ meson production has been also studied by selecting the decay channel $\eta \rightarrow \pi^{+} \pi^{-} \pi^{0}$. After the background subtraction about 600 signal events have been obtained, corresponding to a preliminary cross-section $\sigma\left(e^{+} e^{-} \rightarrow e^{+} e^{-} \eta\right)=(50 \pm 2 \pm 6) \mathrm{pb}$.

The KLOE- 2 data-taking will be mostly at the peak of the $\phi$ resonance, then the presence of the taggers will be essential to suppress the background and to close the kinematics of the events by detecting the scattered leptons. A precision measurement of the $\gamma \gamma \rightarrow \pi^{0} \pi^{0}$ cross-section, to improve the present experimental knowledge in the region of $W_{\gamma \gamma}<800 \mathrm{MeV}$, is planned[2]. Concerning single pseudoscalar final states, the two photon decay width of $\pi^{0}$ and $\eta$ can be measured, as well as the transition form factor, $F_{\pi^{0} \gamma^{*} \gamma^{*}}\left(q_{1}^{2}, q_{2}^{2}\right)$, that is relevant for the calculation of the hadronic light-by-light scattering contribution to $g-2$ of the muon[8].
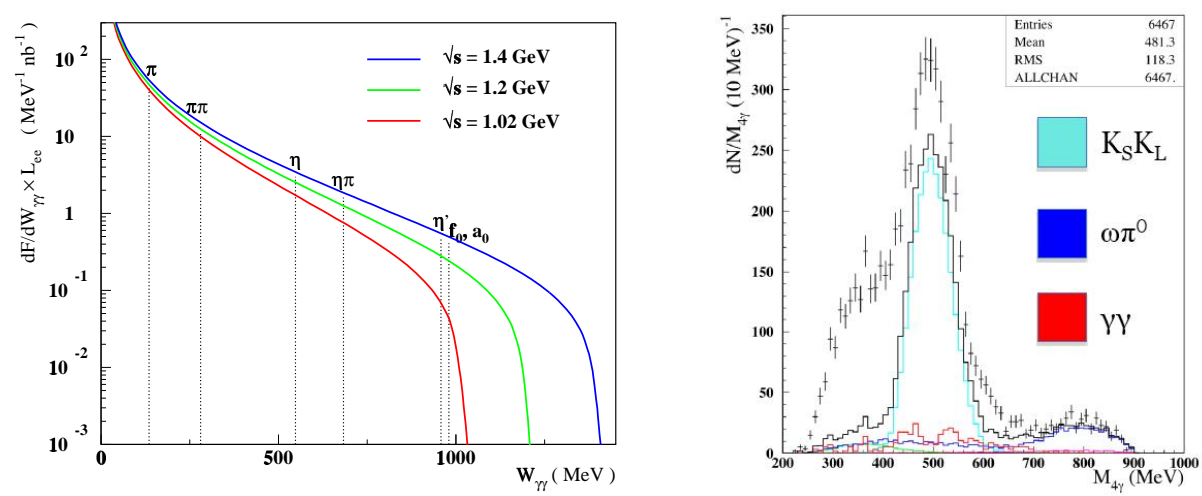

Figure 2: Left: $\gamma \gamma$ flux function for three different center of mass energies. Right: four photon invariant mass distribution.

\section{Hadronic cross-section}

The Standard Model value of the muon magnetic anomaly, $a_{\mu}$, is found to differ about three standard deviations from the experimental value[8]. The main source of uncertainty comes from the hadronic contribution that cannot be calculated in perturbative QCD, but can be evaluated, at lowest order, via a dispersion relation using the measured cross section of $e^{+} e^{-} \rightarrow$ hadrons. The region below $1 \mathrm{GeV}$ is dominated by $e^{+} e^{-} \rightarrow \pi^{+} \pi^{-}$, and can be studied at a $\phi$-factory, operating at fixed $\sqrt{s}$, with the Initial State Radiation method. KLOE has already measured the dipion cross-section 
for $M_{\pi \pi}^{2}$ between 0.35 and $0.95 \mathrm{GeV}^{2}$, by using $e^{+} e^{-} \rightarrow \pi^{+} \pi^{-} \gamma$ events collected at $\sqrt{s}=M_{\phi}$ with the requirement that the photon (not detected) is emitted at small polar angle $\left(\vartheta<15^{\circ}\right)$ with respect to the beamline[9]. In order to extend the $M_{\pi \pi}^{2}$ range down to the dipion threshold, a new measurement has been performed by requiring the photon to be detected at large angle $\left(\vartheta<50^{\circ}\right)$ in the KLOE calorimeter[10]. To reduce the large background coming from $\phi$ decays, the data collected off-peak at $\sqrt{s}=1 \mathrm{GeV}$ have been analyzed. In fig.4 the pion form factor extracted from the large angle measurement is compared to the small angle result. From this measurement an hadronic contribution to the muon anomaly can be evaluated, $a_{\mu}\left(0.1-0.85 \mathrm{GeV}^{2}\right)=(478.5 \pm$ $\left.2.0_{\text {stat }} \pm 4.8_{\text {syst }} \pm 2.9_{\text {theory }}\right) \times 10^{-10}$, which confirms a 3.2 standard deviation discrepancy between the Standard Model and the experimental value of $a_{\mu}$.

At present, work is in progress to obtain in an alternative way the pion form factor from the ratio of the cross-sections $R=\sigma\left(e^{+} e^{-} \rightarrow \pi^{+} \pi^{-} \gamma\right) / \sigma\left(e^{+} e^{-} \rightarrow \mu^{+} \mu^{-} \gamma\right)$, instead of using the absolute normalization to Bhabha scattering events.
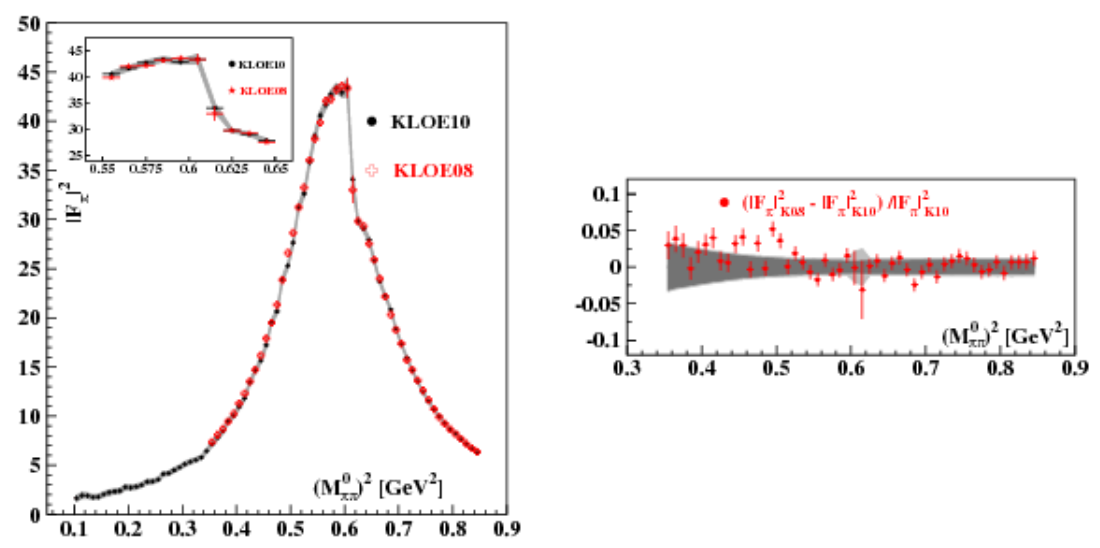

Figure 3: Comparison of the large angle (KLOE10) and small angle (KLOE08) analyses. Left: pion form factor. Right: fractional difference, the gray band is the total error for the KLOE10 measurement.

\section{References}

[1] F.Archilli, these proceedings.

[2] G.Amelino-Camelia et al., Eur.Phys.J C68 (2010) 619.

[3] J.Bijnens, K.Ghorbani, J.High Energy Phys. 11 (2007), 030.

[4] KLOE Collaboration, F.Ambrosino et al., Phys.Lett. B694 (2010) 16.

[5] M.Benayoun et al., Eur.Phys.J. C31 (2003) 525.

[6] D.N.Gao, Mod.Phys.Lett. A17 (2002) 1583.

[7] KLOE Collaboration, F.Ambrosino et al., Phys.Lett. B675 (2009) 283.

[8] F.Jegerlehner, A.Nyffeler, Phys.Rep. 477 (2009) 1.

[9] KLOE Collaboration, F.Ambrosino et al., Phys.Lett. B670 (2009) 285.

[10] KLOE Collaboration, F.Ambrosino et al., arXiv:1006.5313 [hep-ex], submitted to Phys.Lett.B. 